\title{
Update on Dissolution Testing- Recent Activities and Trends
}

\author{
Vivian A. Gray \\ V.A.Gray Consulting, Inc., Hockessin,DE
}

Email for correspondence vagray@rcn.com: www.vagrayconsulting.com

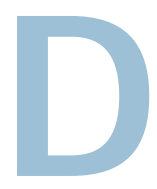

issolution testing is a critical test for measuring the performance of a drug product. The dissolution test is an analytical technique that has undergone significant equipment modifications and improvements spanning the last decade. Dissolution has become an important and widely utilized test receiving more emphasis world wide from regulatory authorities during the last 15 years. It is a critical quality control tool, and an aid to formulation development.Dissolution testing measures change on stability, and is used to establish in-vitro in-vivo correlations for some products. The dissolution test has undergone changes and updates as a result of the increased regulatory interest and the pharmaceutical industry's examination of aspects of dissolution testing to further imp rove and understand the technique.

This article presents the current activities related to dissolution testing in the following organizations or groups:US Pharmacopeia (USP), Food and Drug Administration (FDA), Federation Internationale Pharmaceutique (FIP) Dissolution Working Group, Pharmaceutical Research and Manufacturers of Americas (PhRMA) Dissolution Committee and PhRMA Calibration Subcommittee. Publications and websitessuch as Dissolution Technologies and the Dissolution Discussion Group will be described. A discussion of new technology and trends in dissolution testing is included.

\section{USP}

Over the last 20 years, USP has been actively involved in setting public dissolution standards that have kept up with changes in technology and are responsive to the concerns of drug manufacturers and the FDA.(1)

In 2000,the USP held its Quinquennial meeting. At this five-year meeting the direction of the USP was voted on and set forth through resolutions from the USP convention.USP adopted the following resolutions related to dissolution testing:

- In consultation with the FDA, expand on going harmonization with the European and J apanese Pharmacopeias, and the Pharmacopeias of the Americas.

- Assess the feasibility and advisability of developing approaches for the application of the biopharmaceutics principles in conjunction with appropriate dissolution tests to assure equivalent performances of immediate- and modified-release drug products, taking into account their regulatory control.

- Establish training programs to support appropriate use of USP/NF standards and compendial methods.

To support the first resolution,there is an ongoing effort to harmonize the Dissolution $<711>$, Disintegration $<701>$, and Drug Release $<724>$ General Chapters with the J apanese Pharmacopeia and European Pharmacopeia.This collaboration is part of the $\mathrm{ICH}$ harmonization process and is nearly complete. Aspects of the general chapters, such as adding additional equipment dimensions and specifications, equipment modifications, and allowance of validated sinkers and automated equipment are included in this initiative. Most importantly, however, is the incorporation of the USP Acceptance Criteria. The current debate is over the addition of Stage three testing in the European countries and Britain.

In an initial step toward the second resolution, the new USP Biopharmaceutics Expert Committee has endorsed the adoption of the FDA Biopharmaceutics Classification System (BCS) Guidance. The new guidance on this subject will be put into a new USP General Informational Chapter.

The new USP Biopharmaceutics Expert Committee includes five academicians, four industry representatives, and one member from FDA. The members are Thomas Foster, Pharm.D. (Chair), Gordon Amidon,Ph.D.,Vivian Gray, Michael Mayersohn Ph.D.,Ajaz Hussain Ph.D.,Lewis Leeson Ph.D.,Leon Shargel,Ph.D., Eli Shefter Ph.D., Clarence Ueda,Pharm.D.,Ph.D.,and John Mauger, Ph.D.This committee has decided to update and revise several General Information Chapters and a new general information chapter on dissolution method development and validation was published in the Pharmacopeial Forum in November, 2001 (2).

The third resolution is completed for the area of dissolution.USP now offers a two-day course on dissolution with hands-on training and lectures at the USP headquarters on a monthly basis.

In the area of calibration,the USP has replaced 
the 50-mg Prednisone Calibrator tablet with a 10mg Prednisone tablet.In December of 2000,the ranges of those tablets were changed. For additional information about USP and dissolution calibrator tablets, go to the USP website at www/usp.org.

A Project Team on Calibration has been formed and the initial meeting took place in August 2001. The members of the team are drawn from equipment vendors, USP staff,USP Biopharmaceutics committee, FDA, academia, and the industry. The team has had in-depth discussions on the present calibrator tablets and future directions for calibrator tablets. The second meeting is planned for February 2002.

\section{FDA}

A major contribution of the FDA to dissolution testing has been the numerous guidances issued over the last 10 years. The guidances related to dissolution testing are listed below:

- Dissolution Testing of Immediate Release Solid Oral Dosage Forms

- Extended Release Oral Dosage Forms:Development, Evaluation, and Application of In Vitro/In Vivo Correlations

- Oral Extended (Controlled) Release Dosage Forms In Vivo Bioequivalence and In Vitro Dissolution Testing

- Waiver of In Vivo Bioavailability and Bioequivalence Studies for Immediate-Release Solid Oral Dosage Forms Based on Biopharmaceutics Classification System

- SUPAC-IR:Immediate-Release Solid Oral Dosage Forms:Scale-Up and Post-Approval Changes: Chemistry, Manufacturing, and Controls, In Vitro Dissolution Testing, and In Vivo Bioequivalence Documentation

- SUPAC-MR:Modified-Release Solid Oral Dosage Forms:Scale-Up and Post-Approval Changes: Chemistry, Manufacturing, and Controls, In Vitro Dissolution Testing, and In Vivo Bioequivalence Documentation

In these guidances the FDA presents the similarity factor (the $\mathrm{f}_{2}$ calculation). There have been several discussions on this approach for comparing the dissolution profiles of different batches to assess or establish bioequivalence. (3)
(4) (5).The Biopharmaceutics Classification System, was presented in several guidances, however the definitive guidance - Waiver of In Vivo Bioavailability and Bioequivalence Studies for Immediate-Release Solid Oral Dosage Forms Based on Biopharmaceutics Classification System, shows how this system can use dissolution testing as an alternative to bioequivalence studies.

The FDA appointed a task force, the Gelatin CapsuleWorking Group, to study the in vivo effect of gelatin capsules that show a slowdown in dissolution due to cross-linking. The results were published in the Pharmacopeial Forum. (6) Following the recommendations of the Working Group, the USP Dissolution General Chapter <711> was revised to allow for two-tier dissolution testing. Enzyme can now be added to the dissolution medium and the dissolution test repeated.

The FDA co-sponsors many workshops and conferences on current regulatory issues. The next workshop planned, co-sponsored with AAPS,is in March 2002 on establishing drug product specifications. There will be breakout sessions dealing with several topics, including dissolution and disintegration.

\section{FIP Dissolution Working Group}

This group spearheaded the writing of the FIP Guidelines for Dissolution Testing of Solid Oral Products. The guideline was published as a joint report of the section for Official Laboratories and Medicines Control Services and the section of Industrial Pharmacist of the FIP.(7)

The main focus of the FIP Dissolution Working Group is to provide a forum for discussion of dissolution issues and also provide training for dissolution analysts. The group has sponsored two workshops on dissolution testing of special dosage forms. As a result of these workshops, a review paper was prepared that discusses regulatory aspects and analytical issues relating to special dosage forms (see pages 6-11).

The working group has also held two training workshops on dissolution testing. In 2000, there was an intermediate level workshop in Australia and in J anuary of 2001 there was a basic level workshop held in India (8). The next basic workshop is planned for two cities in China in May 2002. 


\section{Update on Dissolution Testing ... continued}

\section{PhRMA Dissolution Committee}

One major function of this committee was to conduct a collaborative study that determines the ranges for USP Calibrator Tablets.A recent collaborative study includes 20-30 laboratories that perform dissolution tests on the calibrator tablets using both the basket and paddle dissolution apparatus at different speeds. The studies are published in the Pharmacopeial Forum of the USP to inform the scientific community how the range specifications are obtained and show the detailed statistical analysis (9). With the most recent lot of Prednisone Tablets, the USP has managed the collaborative study with cooperation and participation of PhRMA.

This PhRMA committee routinely polls its members for opinions on regulatory policies. For example, members of the committee examined the harmonization proposals for the Dissolution and Disintegration general chapters.

\section{PhRMA Dissolution Calibration Subcommittee}

Within the PhRMA Dissolution Committee is a Dissolution Calibration Subcommittee.This subcommittee's purpose is to examine dissolution apparatus calibration and look for ways to reduce testing without compromising the standards for equipment evaluation. Mechanical calibration was studied thoroughly as an alternative to using USP calibrator tablet testing. (8) The PhRMA Dissolution Calibration Subcommittee is exploring other ways of examining the utility of calibrator testing.A survey to gather historical calibrator data is now available through several websites.

\section{Dissolution Technologies}

This publication is published quarterly, providing useful information on dissolution testing. It provides a forum for dissolution analysts to receive and exchange information on various dissolution topics. The Dissolution Technologies website can be accessed at www.dissolutiontech.com.

\section{Dissolution Discussion Group (DDG)}

This group has an Internet discussion site at www.dissolution.com.It provides an interactive bulletin board forum on all topics in dissolution testing. The group meets annually where round table discussions are conducted on a variety of topics and issues. Regional meetings are also sponsored in various locations in the US and around the world.

\section{New Dissolution Technology}

The areas of most recent growth in dissolution testing equipment are fiber optic technology and fully automated dissolution testers.

The use of fiber optic technology as a labor saving feature of testing has been discussed for years. Now there are at least three major vendors selling this equipment in a variety of configurations. Several articles on the application and validation of fiber optics technology and its utility have been published.(11) (12) (13) (14) The fiber optics detection cell is either located in the basket or paddle shaft or as a probe placed directly into the medium. Fiber optic detection avoids the filtering and pumping of sample and permits continuous measurements during the course of the dissolution test.

Automation has become more sophisticated with on-line UV measurement systems that use flow cells for real-time determinations, eliminating fraction collection and separate UV analysis. There are fully automated systems that deaerate and heat medium, fill the vessels with medium,drop tablets, filter and sample the vessel medium,and clean the vessels.

Other labor saving products, such as premixed medium and mobile phase, are now commercially available. Premixed media of all compositions, and in some cases deaerated, is shipped in sealed plastic containers and offers off-the-shelf convenience.

\section{Conclusion}

Dissolution testing is continuing to grow and mature as a critical technology to pharmaceutical analysis. The resources for information are abundant and many organizations are actively involved in the improvement and application of the technique. The manufacturers of dissolution equipment are actively developing new and more sophisticated equipment. Worldwide regulatory agencies are relying on the test more and more for relevance to in vivo performance. It is anticipated that dissolution will continue to grow as a meaningful and sophisticated test for the analysis of pharmaceutical dosage forms.

\section{References}

1.Gray VA, Grady LT.USP Dissolution StandardsRevisions, Initiative, and New Policies Since 1980, Pharm Forum 1997:23 (3):4183-4197. 2. Gray VA, Brown CK,Dressman J B, Leeson LJ .A 